\title{
Effectiveness of mobile-phone short message service (SMS) reminders for ophthalmology outpatient appointments: Observational study
} Elizabeth Koshy*, Josip Car and Azeem Majeed

Address: Department of Primary Care and Social Medicine, Imperial College London, London, UK

Email: Elizabeth Koshy* - e.koshy@imperial.ac.uk; Josip Car - j.car@imperial.ac.uk; Azeem Majeed - a.majeed@imperial.ac.uk

* Corresponding author

Published: 3I May 2008

BMC Ophthalmology 2008, 8:9 doi:10.1186/147|-2415-8-9

This article is available from: http://www.biomedcentral.com/I47I-24/5/8/9

(C) 2008 Koshy et al; licensee BioMed Central Ltd.

This is an Open Access article distributed under the terms of the Creative Commons Attribution License (http://creativecommons.org/licenses/by/2.0), which permits unrestricted use, distribution, and reproduction in any medium, provided the original work is properly cited.

\begin{abstract}
Background: Non-attendance for hospital outpatient appointments is a significant problem in many countries. It causes suboptimal use of clinical and administrative staff and financial losses, as well as longer waiting times. The use of Short Message Service (SMS) appointment reminders potentially offers a cost-effective and time-efficient strategy to decrease non-attendance and so improve the efficiency of outpatient healthcare delivery.
\end{abstract}

Methods: An SMS text message was sent to patients with scheduled appointments between April and September 2006 in a hospital ophthalmology department in London, reminding them of their appointments. This group acted as the intervention group. Controls were patients with scheduled ophthalmology appointments who did not receive an SMS or any alternative reminder.

Results: During the period of the study, II.2\% (50/447) of patients who received an SMS appointment reminder were non-attenders, compared to I8.1\% (I720/95I2) who did not receive an SMS reminder. Non-attendance rates were $38 \%$ lower in patients who received an SMS reminder than in patients who did not receive a reminder ( $R R$ of non-attendance $=0.62 ; 95 \% \mathrm{Cl}=$ $0.48-0.80)$.

Conclusion: The use of SMS reminders for ophthalmology outpatient appointments was associated with a reduction of $38 \%$ in the likelihood of patients not attending their appointments, compared to no appointment reminder. The use of SMS reminders may also be more cost-effective than traditional appointment reminders and require less labour. These findings should be confirmed with a more rigorous study design before a wider roll-out.

\section{Background}

Non-attendance for hospital outpatient appointments is a major burden on healthcare systems and costs the National Health Service (NHS) in the UK an estimated $£ 790$ million per year [1]. It reduces the efficiency and effectiveness of the delivery of outpatient healthcare and causes substantial financial losses for healthcare systems
[2]. It also results in suboptimal use of clinical and administrative staff and results in increased waiting times for other patients [3]. The increased waiting time can result in delay in presentation of patients' symptoms and also decreased monitoring of long-term chronic conditions; which can, in turn, lead to increased patient morbidity 
[4]. Additionally, there are worse outcomes for nonattenders and a loss of continuity of care [5].

Some of the main reasons for patients not attending their outpatient appointments are forgetting their appointments $[6]$ and confusion over the date, time and location of the appointment $[6,7]$ There are many methods of delivering appointment reminders which have been studied to date, including personalised and automated telephone reminders $[6,8-12]$, posted letters $[13,9]$ and awareness campaigns [14]. Most of the studies have shown a reduction in non-attendance rates, irrespective of the method of reminder used. However, there is a paucity of research in relation to the use of mobile phone SMS (Short Message Service)/text message reminders for outpatient hospital appointments [3].

As mobile phone ownership continues to increase rapidly in many countries worldwide, there is potential to utilise SMS reminders to increase the effectiveness and efficiency of health care delivery. A recent survey showed that over $85 \%$ of adults in the UK used a mobile phone [15] SMS facilities on mobile phones were introduced in the early 1990 s and now represents one of the most widely used methods of communication, with about 41.8 billion texts sent in the UK in 2006 [16]. SMS messages have a number of characteristics that make them very appropriate for use in a healthcare setting including: direct patient communication, privacy, confidentiality, swift delivery of messages and receipt of responses, convenience for health providers and patients. SMS messaging technology also allows the dispatching of substantial numbers of messages simultaneously, so reducing labour expenditure.

Non-attendance at hospital outpatient clinics is a common problem which every specialty faces. It is particularly important for specialties such as ophthalmology which are predominantly outpatient-based [17] An audit in an ophthalmology clinic in a Birmingham hospital in the UK, reported a non-attendance rate of $12.6 \%$ [18]. A New Zealand study found non-attendance rates at a public eye clinic of $17.2 \%$ [17]. This highlights that it is essential to maximise patient attendance to optimise the efficiency of the service delivered. We could not identify any studies relating to the use of SMS-based reminders for hospital appointments for ophthalmology appointments within the Medline, Embase, Cochrane, and PsycInfo databases.

This pilot study primarily focused on patients attending ophthalmology clinics as these outpatient clinics had one of the highest non-attendance rates. Our study assessed the effectiveness of the use of SMS-based reminders for hospital outpatient appointments as a method of reducing the non-attendance rates in an inner-city London teaching hospital. The null hypothesis was that (non- )attendance rates for those receiving an SMS reminder did not differ from the (non-)attendance rates for those who did not receive an SMS reminder.

\section{Methods}

This is an observational study and analysis is based on data collected from the ophthalmology department at Barts and the London Hospitals NHS Trust, UK between April and September 2006. Ethics committee approval was obtained from Barts and The London NHS Trust for SMS reminders to be sent to patients. We analysed data that did not contain any patient identifiable information.

All patients, where a mobile number was obtainable from the Patient Administration System and who were due to attend their first (new) ophthalmology consultation during the study period, were sent an automated SMS reminder. The SMS reminders were sent one day before the appointment if it was booked within seven days of the appointment. If an appointment had been booked more than seven days in advance of an appointment, the patient received an SMS four days beforehand. A four day interval was chosen to minimise the time delay for patients to still forget their appointments, but also to allow time to reschedule appointments, if patients subsequently cancelled their appointment. Information required for the SMS reminders was obtained from the Patient Administration System hospital database. This included the patients' mobile numbers, appointment dates and times. Patients who received an SMS formed the intervention group. A control group were all patients who had a scheduled first (new) appointment during this same study period, who may or may not have had a mobile phone, but whose mobile number was not available and did not receive any other form of appointment reminder.

The text message read: "This is a reminder of your appointment at Barts and the London Hospital at <time $><$ date>. Please call xxxxx or reply to text to cancel."

The automated SMS reminders transmitted were timed so that they did not reach the recipients at inappropriate times, such as night-time. The attendance/non-attendance status for patients who received and did not receive an SMS appointment reminder were recorded using the IT software installed. Data on cancellations by patients for the SMS and control groups was also collected.

Statistical analysis was performed using Stata 9. The attendance rates in the SMS reminder group were compared with those in the control group. A chi-squared test was conducted to compare the proportions of patients not attending appointments and also the proportions of cancellations by patients between the SMS and the control 
groups. Relative risks of non-attendance are presented with $95 \%$ confidence intervals (CI).

\section{Results}

Of the 9959 ophthalmology hospital outpatient appointments between April and September 2006, 447 patients $(4.5 \%)$ received an SMS appointment reminder (Table 1$)$. The control group (patients who did not receive an SMS reminder) consisted of 9512 patients and had a nonattendance rate of $18.1 \%$ (1720/9512). Amongst the patients who received an SMS reminder (447 patients), the non-attendance rate was 11.2\% (50/447) (Table 1, Fig 1 ). The absolute reduction in non-attendance rates for those who received an SMS reminder was 6.9\%. This represents about 31 more (of the 447 ophthalmology outpatient appointments) being kept over a 6 month period that would have otherwise been lost through patients not keeping their scheduled appointments. The cancellation rates of appointments by patients who received an SMS and those who did not receive an SMS were $13.4 \%$ and $11.2 \%$, respectively (Table 1 ).

Patients who received a text reminder were $38 \%$ less likely to be non-attenders for their appointment (relative risk of non-attendance $=0.62 ; 95 \%$ CI $0.48-0.80, \mathrm{p}=0.0002$ ) . Patients in the SMS group were also $26 \%$ more likely to cancel their appointments, but this was not statistically significant (relative risk of cancellation 1.26, 95\% confidence interval $0.98-1.61, \mathrm{P}=0.068$ ). There was a total of 340,700 scheduled outpatient appointments (for all specialities) between April-September 2006. Of the 340,146 who did not receive an SMS appointment reminder the non-attendance rate was $12.1 \%$.

The cost of sending an automated SMS reminder was 7.2 pence. Therefore, extrapolating the six month data to one year for the ophthalmology clinics, sending 894 SMS reminders $(447 \times 2)$ would have cost $\mathfrak{E} 64.37$. The cost of approximately $62(31 \times 2)$ extra appointments being attended as a consequence of the SMS reminders, is $£ 4030$ (with a cost of a hospital appointment in England being approximately $£ 65.00$ ). Therefore, the net saving would

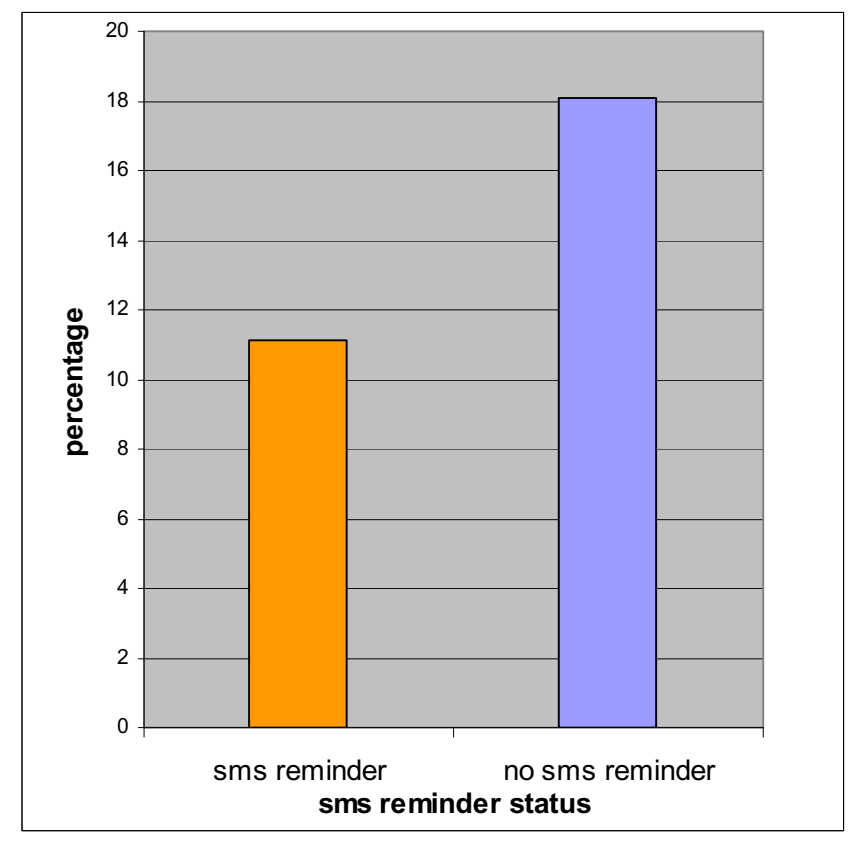

Figure I

Percentage of non-attenders by SMS reminder status.

have been $£ 3965.63$, just for the ophthalmology department. The 'number needed to text' (NNT) in this study to prevent one appointment non-attendance is 14 (95\% confidence interval: 10 to 31 ).

\section{Discussion}

Patients who had received an SMS reminder were significantly more likely to attend their ophthalmology hospital appointments compared to those who had not received an SMS. This study showed that sending SMS reminders led to a $6.9 \%$ absolute reduction and $38 \%$ relative reduction in non-attendance rates for ophthalmology outpatient appointments. Non-attendance rates in the SMS group and the control group were $11.2 \%$ and $18.1 \%$, respectively. The cancellation of appointments in the SMS group compared to the control group was slightly higher

Table I: Non-attendance rates for patients sent a SMS reminder and those not sent a SMS reminder

\begin{tabular}{lllllll}
\hline SMS appointment reminders & & No SMS appointment reminders & $\begin{array}{c}\text { Non-attendance } \\
\text { rate reduction } \\
\text { (\%) } \begin{array}{l}\text { with SMS } \\
\text { reminders }\end{array}\end{array}$ \\
\hline $\begin{array}{l}\text { TOTAL } \\
\text { outpatient } \\
\text { appointments }\end{array}$ & $\begin{array}{l}\text { Non-attendance } \\
\text { appointment } \\
\text { numbers (\%) }\end{array}$ & $\begin{array}{l}\text { Cancellations by } \\
\text { patients (\%) }\end{array}$ & $\begin{array}{l}\text { TOTAL } \\
\text { outpatient } \\
\text { appointments }\end{array}$ & $\begin{array}{l}\text { Non-attendance } \\
\text { appointment } \\
\text { numbers (\%) }\end{array}$ & $\begin{array}{l}\text { Cancellations by } \\
\text { patients (\%) }\end{array}$ \\
\hline 447 & $50(11.2)$ & $60(13.4)$ & 9512 & $1720(18.1)$ & $1016(10.7)$ \\
\hline
\end{tabular}

$N=9959$ 
$(13.4 \%$ vs $11.2 \%)$. Cancellation rates were not statistically significantly different between the two groups but there was a trend towards a higher cancellation rate in the SMS group (relative risk of cancellation 1.26; 95\% CI 0.98 1.61).

The non-attendance rate for ophthalmology appointments $(18.1 \%)$ in our control group was higher than the mean non-attendance rate for all the different hospital specialty outpatient appointments $(12.1 \%)$. This confirmed that non-attendance was a greater problem for the ophthalmology department compared to many other specialties within the same hospital. It is difficult to speculate why this might be, but there could have been administration problems, for example, due to staff shortages; or perhaps, though this may be unlikely, the demographic characteristics or behaviours of the patients in this geographical area who attend the ophthalmology clinics may differ from that of other specialties. The $18.1 \%$ nonattendance rate in the control group in our study is higher than that quoted in other studies $[19,18,17]$. For example, the Birmingham ophthalmology department audit, over a year, was $12.6 \%$ [18]. However, our study was only conducted over a 6 month period and ideally, information relating to a year, taking into account seasonal variations, would be valuable. Also, this Birmingham study was in 1990-1, so non-attendance rates may have changed over this time period. A New Zealand audit showed a nonattendance rate of $17.2 \%$ [17].

The non-attendance rate of the SMS group in our study was $38 \%$ lower than that of the control group $(R R=0.62)$ and there was strong evidence $(P=0.0002)$ to reject the null hypothesis of no difference between these two groups. A multi-centred, randomised controlled trial in China also found an increase in the likelihood of attendance $(\mathrm{OR}=1.59, \mathrm{P}=0.005)$ [20]. This study had 993 participants who were divided into 3 study arms; and compared SMS reminders and mobile telephone conversations reminders with a control group of no intervention. There was no statistically significant difference in nonattendance observed between their SMS group and mobile phone-call reminders. This study was conducted in a primary care setting, which may have represented a systematically different group of patients in terms of nonattendance characteristics to those attending secondary care appointments, so direct comparisons cannot be made. Additionally, Family Practice-based factors such as mistakes or misunderstandings surrounding appointment details, relayed over the telephone, from the health provider are possible reasons for non-attendance in this healthcare setting [21].

The percentage reduction in non-attendance rates with SMS reminders of $6.9 \%$ in our study is lower than that reported for a recent study in an Irish ENT outpatient department [2], where there was a $11.6 \%$ reduction (from $33.6 \%$ to $22 \%$ ). This study, however, used data from a much larger sample size (3981 patients) and covered a 3 year period. Studies of other methods of appointment reminders such as posted letters and telephone calls found reductions of non-attendance rates between $6 \%$ and $19 \%[6,8,12,10]$. The reduction in non-attendance found in our study is within the range of these other studies. Therefore, SMS reminders are at least as effective as alternative methods. The SMS reminders also have the advantage of being more cost-effective and requiring less labour than the other methods.

\section{Limitations of this study}

Although there seems to be an association between the use of SMS reminders and a decrease in non-attendance rates, this is not necessarily causal and there could be confounding factors and biases which may partially or fully explain this association. This study suggests that the use of SMS reminders could help to reduce ophthalmology nonattendance rates. However, the design in this paper is suboptimal, and a rigorous randomised controlled trial, stratified by socio-demographic characteristics and clinical conditions, is required to determine if the potential benefits observed in this study can be replicated. Knowledge of the patients' clinical presentation in the intervention and control groups is also important as it could affect the 'value' placed on it by patients and potentially affect the likelihood of an appointment being kept.

We do not know what proportion of the control group owned mobile phones, but whose mobile number was not available for this service. We are also unaware of the number of patients who were unfamiliar with using textmessaging and so unable to read the reminder. There could also have been participation bias, as the SMS group patients (who provided a mobile phone number) may have been a more motivated group of patients; and these patients may have been more likely to attend their appointments irrespective of receiving an SMS reminder. This could have potentially led to an over-estimate of the association between SMS reminders and non-attendance rates. Data was only available for a 6 month period, and so did not take account of seasonal and monthly variations in non-attendance. However, using the same timeframe for the controls helped to reduce the chance of monthly and seasonal variations contributing to the observed differences. We did not have demographic information relating to those patients who owned a mobile phone or for those who received an SMS reminder who subsequently attended and did not attend their scheduled appointments. Data relating to potential confounding factors (such as age, sex, ethnicity and socio-economic status) was not collected, so we were not able to adjust for 
these in the statistical analysis. We did not have demographic information available relating to age in either groups and recognise this is a weakness and could account, in part or fully, for the observed difference. However, as younger patients are more likely to own mobile phones and also have higher non-attendance rates, the fact that the non-attendance rate decreased in the SMS group (who are more likely to be younger), suggests that use of SMS reminders may be beneficial to target younger patients. Finally, patients who received SMS reminders were for a first consultation and it has been suggested that follow-up appointments have higher non-attendance rates [22]. This was the first stage of the study; the next stage will be focusing on follow-up appointments.

\section{What this study adds}

SMS appointment reminders seem to be an effective and efficient method of improving ophthalmology outpatient attendance, which is less labour intensive than the more traditional reminder systems that have been used in the past. The facility for patients to reply or call in response to an SMS reminder to cancel their appointment can help to reduce the non-attendance rates and free up appointments for other patients, which otherwise may be lost. We cannot extrapolate our findings to other specialties or hospitals; so future research should include suitably powered randomised controlled trials for assessing SMS appointment reminders for ophthalmology and other specialties to assess the cost-effectiveness within the NHS. The use of SMS technology is expanding and has already been used to help in the management of some health problems and diseases; for example, asthma [23] and diabetes [24,25] management and smoking cessation [26]. Hence, the potential scope for the further utilisation of this technology is tremendous. SMS reminders could offer a cost-effective method for reducing non-attendance rates for retinal screening appointment follow-ups for diabetic patients.

\section{Strengths and weaknesses of SMS technology}

In addition to the advantages already described, SMS reminders require minimal investment in IT infrastructure, as this is already in existence; as the IT software for sending automated SMS integrates with existing electronic patient health records and hospital administrative database systems. Once the system is in place, the cost of running the service increases very little as the number of SMS reminders increases. As they are automated messages, it does not require staff training so this offers a time, labour and cost-efficient system.

There are a number of potential weaknesses. Patients may not receive the SMS reminders due to incorrect data entry. However, this problem can occur with other methods of appointment reminders such as letters and telephone calls (with change of address) [12]. The uses of different tech- nologies are very fluid and dynamic and so it is not known how the use of mobile phones and the use of SMS technology will evolve over time. However, it is anticipated that there will be an increase in mobile phone ownership and use of SMS messaging facilities in the immediate few years. Elderly people have lower ownership rates of mobile phones and may not be able to use SMS facilities [27]. The Office for National Statistics (UK) [27] found mobile ownership in 2003 varied by age, with nearly $90 \%$ of 15-24 year olds owning one and less than a quarter of those aged 75 years and over owning one in 2003. However, between 2001 and 2003, the largest increases in ownership were amongst those aged 75 and over, with the proportions almost doubling [27]. However, it has traditionally been younger patients who have missed their outpatient appointments more than older patients, so the use of SMS reminders could be targeted towards patients under 60 years old.

\section{Cost-effectiveness analysis}

There is always some wastage in any system and the real cost of a missed appointment is difficult to quantify. It may be, in some cases, that the appointment may not have really been needed, although this is difficult to prove. For example, patients' agenda may influence presentation and attendance [28]. Based on the 'number needed to text' analysis, approximately fourteen people would need to be sent an SMS reminder to prevent one non-attendance. As the cost of outpatient appointments is considerable, this could potentially be worthwhile, but needs to be tested through a more rigorous economic analysis. A well-designed randomised controlled trial would give a more accurate representation of the NNT to prevent one non-attendance. Only around 5\% of the patients with scheduled ophthalmology appointments were sent SMS reminders in this study; so, if the reminders were sent on a much larger scale to patients with first and follow-up appointments and for all the departments in the hospital, the savings to the NHS could be large. A previous cost-effectiveness analysis has estimated that the annual direct cost of missed hospital appointments in England is estimated to be close to $£ 575$ million [1] and that the use of SMS-based reminders could lead to a potential saving of $£ 55.6$ to $£ 83.5$ million a year [1], so the potential scope for this technology is considerable.

\section{Conclusion}

The use of SMS reminders for ophthalmology outpatient appointments was associated with a reduction of $38 \%$ in the likelihood of patients not attending their appointments, compared to no appointment reminder. Additionally, the use of SMS reminders appears more cost-effective than traditional appointment reminders and requires less labour. These findings suggest that SMS text reminders have great potential value in reducing non-attendance 
rates in outpatient departments but that the potential benefits should be confirmed with a more rigorous study design before a wider roll-out.

\section{Competing interests}

The authors declare that they have no competing interests.

\section{Authors' contributions}

EK and JC conceived the idea for this paper. EK wrote the manuscript. EK and AM performed the statistical analysis and data interpretation. JC and AM were involved in interpretation of findings and revised the paper critically for intellectual content. All authors read and approved the final manuscript. EK and JC are the guarantors for this study.

\section{Acknowledgements}

We would like to acknowledge Barts and the London Hospitals NHS Trust who were responsible for initiating this intervention study. We would also like to thank Tobias Alpsten, the Managing Director of iPLATO. iPLATO is the telecommunications company, who integrated the patient information systems and were responsible for organising all the automated SMS appointment reminders to patients at Barts and The London Hospitals NHS Trust.

We would also like to thank the Department of Health (UK) National Institute for Health Research who funded a research fellowship for Dr Elizabeth Koshy. The Department of Primary Care \& Social Medicine at Imperial College receives support from the NIHR Biomedical Research Centre Funding Scheme.

\section{References}

I. Atun AR SSR Mohan A.: Uses and Benefits of SMS in Healthcare Delivery. Centre for Health Management. Tanaka Business School. Imperial College London . 2005.

2. Geraghty M, Glynn F, Amin M, Kinsella J: Patient mobile telephone 'text' reminder: a novel way to reduce non-attendance at the ENT out-patient clinic. J Laryngol Otol 2007: I-3.

3. Downer SR, Meara JG, Da Costa AC: Use of SMS text messaging to improve outpatient attendance. Med J Aust 2005, 183(7):366-368.

4. Murray M: Patient care: access. BMJ 2000, 320(7249): $1594-1596$.

5. Karter AJ, Parker MM, Moffet HH, Ahmed AT, Ferrara A, Liu JY, Selby JV: Missed appointments and poor glycemic control: an opportunity to identify high-risk diabetic patients. Med Care 2004, 42(2): II0-I I5.

6. Sawyer SM, Zalan A, Bond LM: Telephone reminders improve adolescent clinic attendance: a randomized controlled trial. J Paediatr Child Health 2002, 38( I):79-83.

7. NHS National Research Register [https://portal.nihr.ac.uk/ Pages/NRRArchiveSearch.aspx]

8. Reekie $D$, Devlin $\mathrm{H}$ : Preventing failed appointments in general dental practice: a comparison of reminder methods. Br Dent J 1998, 185(9):472-474.

9. Lieu TA, Capra AM, Makol J, Black SB, Shinefield HR: Effectiveness and cost-effectiveness of letters, automated telephone messages, or both for underimmunized children in a health maintenance organization. Pediatrics 1998, I0I(4): E3.

10. Reti S: Improving outpatient department efficiency: a randomized controlled trial comparing hospital and generalpractice telephone reminders. N Z Med J 2003, I I 6( I I 75): U458.

II. Lee CS, McCormick PA: Telephone reminders to reduce nonattendance rate for endoscopy. J R Soc Med 2003 96(II):547-548
12. Hashim MJ, Franks P, Fiscella K: Effectiveness of telephone reminders in improving rate of appointments kept at an outpatient clinic: a randomized controlled trial. J Am Board Fam Pract 200I, I4(3):193-196.

13. Tierney CD, Yusuf H, McMahon SR, Rusinak D, MA OB, Massoudi MS, Lieu TA: Adoption of reminder and recall messages for immunizations by pediatricians and public health clinics. Pediatrics 2003, I I 2(5): I076- 082.

14. Developing Patient Partnerships. Public and practices unite against missed appointments [http://www.dpp.org.uk/en/l/ missedappointments.qxml]

15. Self A ZL: Office for National Statistics. Social Trends http:// www.statistics.gov.uk/downloads/theme_social/ Social_Trends37/Social_Trends_37.pdf. 2007.

16. The Mobile Data Association (online). Latest Text and Mobile Internet Page Impression Statistics. [http:// www.themda.org/PressReleases/

Page Press PressReleases LatestStats.asp]

17. Koppens JM, Dai S, Mora J: Factors related to non-attendance in a public eye clinic. Clin Experiment Ophthalmol 2005, 33(5):553-554.

18. King A, David D, Jones HS, O'Brien C: Factors affecting nonattendance in an ophthalmic outpatient department. J $R$ Soc Med 1995, 88(2):88-90.

19. Potamitis T, Chell PB, Jones HS, Murray PI: Non-attendance at ophthalmology outpatient clinics. I $R$ Soc Med 1994 87(10):59I-593.

20. Leong KC, Chen WS, Leong KW, Mastura I, Mimi O, Sheikh MA, Zailinawati $\mathrm{AH}, \mathrm{Ng} \mathrm{CJ}$, Phua $\mathrm{KL}$, Teng $\mathrm{CL}$ : The use of text messaging to improve attendance in primary care: a randomized controlled trial. Fam Pract 2006, 23(6):699-705

2I. Neal RD, Hussain-Gambles M, Allgar VL, Lawlor DA, Dempsey O: Reasons for and consequences of missed appointments in general practice in the UK: questionnaire survey and prospective review of medical records. BMC Fam Pract 2005, 6:47.

22. Milne RG, Horne M, Torsney $B$ : SMS reminders in the UK national health service: an evaluation of its impact on "noshows" at hospital out-patient clinics. Health Care Manage Rev 2006, 3 I (2): 130-136.

23. Ostojic V, Cvoriscec B, Ostojic SB, Reznikoff D, Stipic-Markovic A Tudjman Z: Improving asthma control through telemedicine: a study of short-message service. Telemed J E Health 2005, I I ( I):28-35.

24. Yoon KH, Kim HS: A short message service by cellular phone in type $\mathbf{2}$ diabetic patients for $\mathbf{2}$ months. Diabetes Res Clin Pract 2007, 79:256-6I.

25. Kim HS, Jeong HS: A nurse short message service by cellular phone in type-2 diabetic patients for six months. J Clin Nurs 2007, 16(6): 1082-1087

26. Rodgers A, Corbett T, Bramley D, Riddell T, Wills M, Lin RB, Jones M: Do u smoke after txt? Results of a randomised trial of smoking cessation using mobile phone text messaging. Tob Control 2005, 14(4):255-26I.

27. Office for National Statistics (ONS) 2003 [http://www.statis tics.gov.uk/STATBASE/ssdataset.asp?vlnk $=7202 \& M o r e=Y]$

28. Hamilton $\mathrm{W}$, Britten $\mathrm{N}$ : Patient agendas in primary care. $\mathrm{Bmj}$ 2006, 332(7552): $1225-1226$

\section{Pre-publication history}

The pre-publication history for this paper can be accessed here:

http://www.biomedcentral.com/1471-2415/8/9/prepub 